\title{
Welcome to a new-born Frontiers of Chemistry in China
}

\author{
Yuliang YANG (两)
}

AS Editor-in-Chief of Frontiers of Chemistry in China (FCC), it is my great pleasure to welcome you to the first issue of FCC of 2010.

In China, the research of chemical science in almost every area has been growing ever more rapidly in recent years. The world has witnessed the astonishing progress achieved by Chinese scientists. However, the development of Chinese chemical journals does not yet match the progress in research field. It becomes urgent for us to establish a China-based journal that publishes important, primary research articles for a global readership.

Toward this end we launched the new-born Frontiers of Chemistry in China. The goal of quarterly publication is to disseminate original, peer-

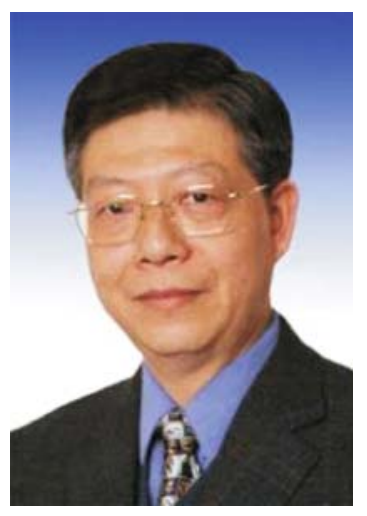
reviewed feature papers and research works of high quality to the international community through efficient publication. It is our firm belief that the growing strength of research in China will further benefit from the many publications in subsequent issues of the journal. I hope you find the journal providing research essential to reflecting up-to-date progresses covering all areas of chemistry.

I would like to acknowledge all those who have worked hard for FCC by participating in the new Editorial Board, by inviting and submitting manuscripts, by being peer-reviewers, by providing necessary support, and by offering valuable advice. We call upon more researchers to join us in support of Frontiers of Chemistry in China, and to help building it into an advanced and successful journal.

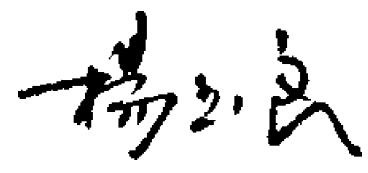

Yuliang YANG

Editor-in-Chief

Frontiers of Chemistry in China

Yuliang YANG

Fudan University, Shanghai 200433, China

E-mail: yangyl@fudan.edu.cn 